\title{
Supporting Information: Quantifying the Composition of Methylammonium Lead Iodide Perovskite Thin Films with Infrared Spectroscopy
}

Xiaokun Huang ${ }^{1,2,3 \#, ~ M i c h a e l ~ S e n d n e r ~}{ }^{1,3 \#}$, Christian Müller ${ }^{1,2}$, Michele Sessolo $^{4}$, Lidón GilEscrig, 9 , Wolfgang Kowalsky,1,2, Annemarie Pucci ${ }^{1,3}$, Sebastian Beck ${ }^{1,3}$, and Robert Lovrinčićl ${ }^{1,+}$

${ }^{1}$ InnovationLab, Heidelberg, Germany

${ }^{2}$ Institute for High-Frequency Technology, Braunschweig Technical University, Germany

${ }^{3}$ Kirchhoff Institute for Physics, University of Heidelberg, Germany

${ }^{4}$ Instituto de Ciencia Molecular, Universidad de Valencia, C/Catedrático J. Beltrán 2, 46980

Paterna, Spain

๑New affiliation: Helmholtz-Zentrum Berlin, 12489 Berlin, Germany

${ }^{+}$New affiliation: trinamiX GmbH, Ludwigshafen, Germany

AUTHOR INFORMATION

\# These authors contributed equally to this work.

Corresponding Author

*E-mail: r.lovrincic@tu-braunschweig.de 


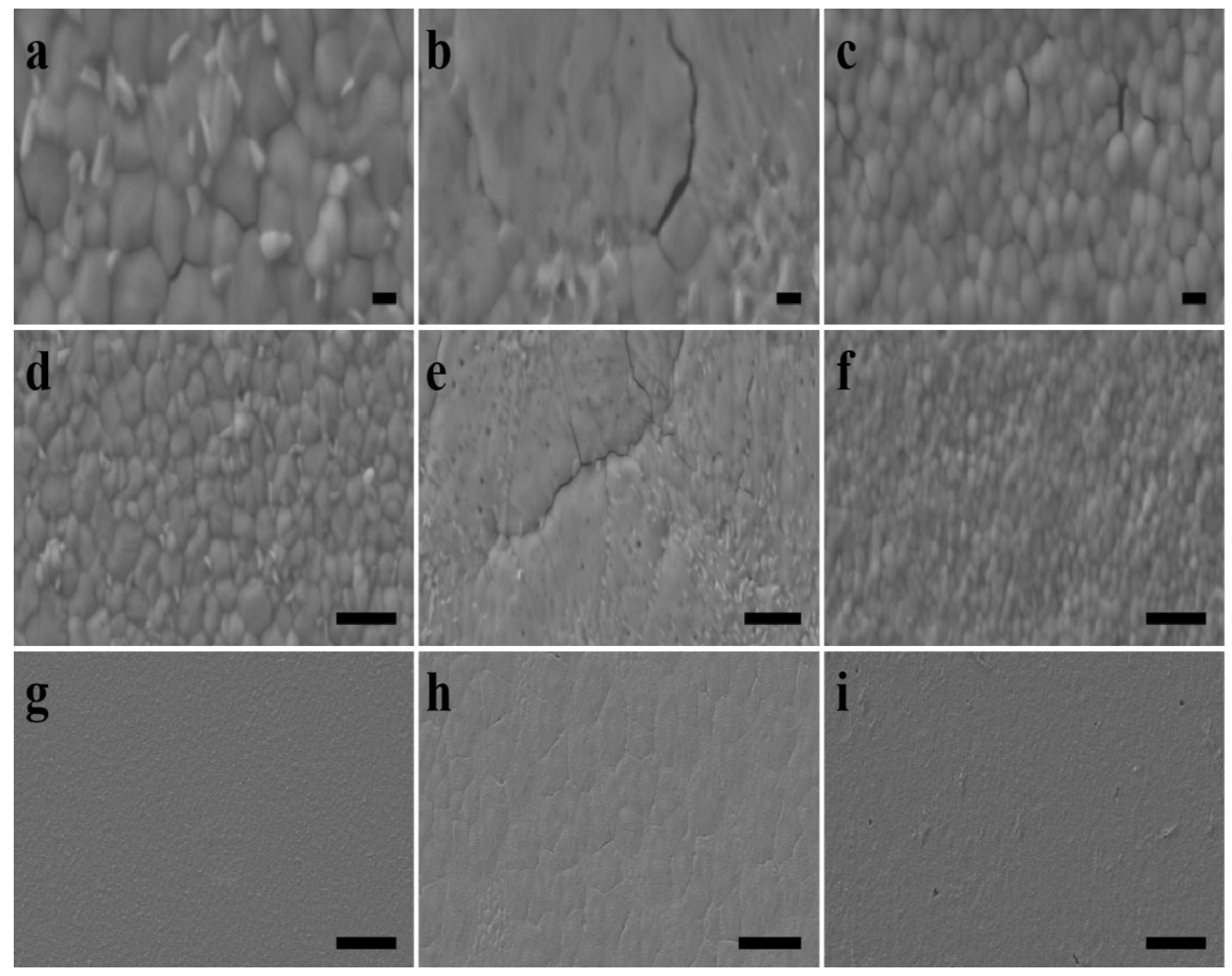

Figure S1: SEM images of solution processed $\mathrm{MAPbI}_{3}$ thin films on silicon with varying MA content in the precursor solution: (a)0.8M MA, (b)1.0M MA, (c)1.2M MA, scale bar: 200nm; (d)0.8M MA, (e)1.0M MA, (f)1.2M MA, scale bar: $1 \mu \mathrm{m}$; (g)0.8M MA, (h)1.0M MA, (i)1.2M MA, scale bar: $10 \mu \mathrm{m}$. 


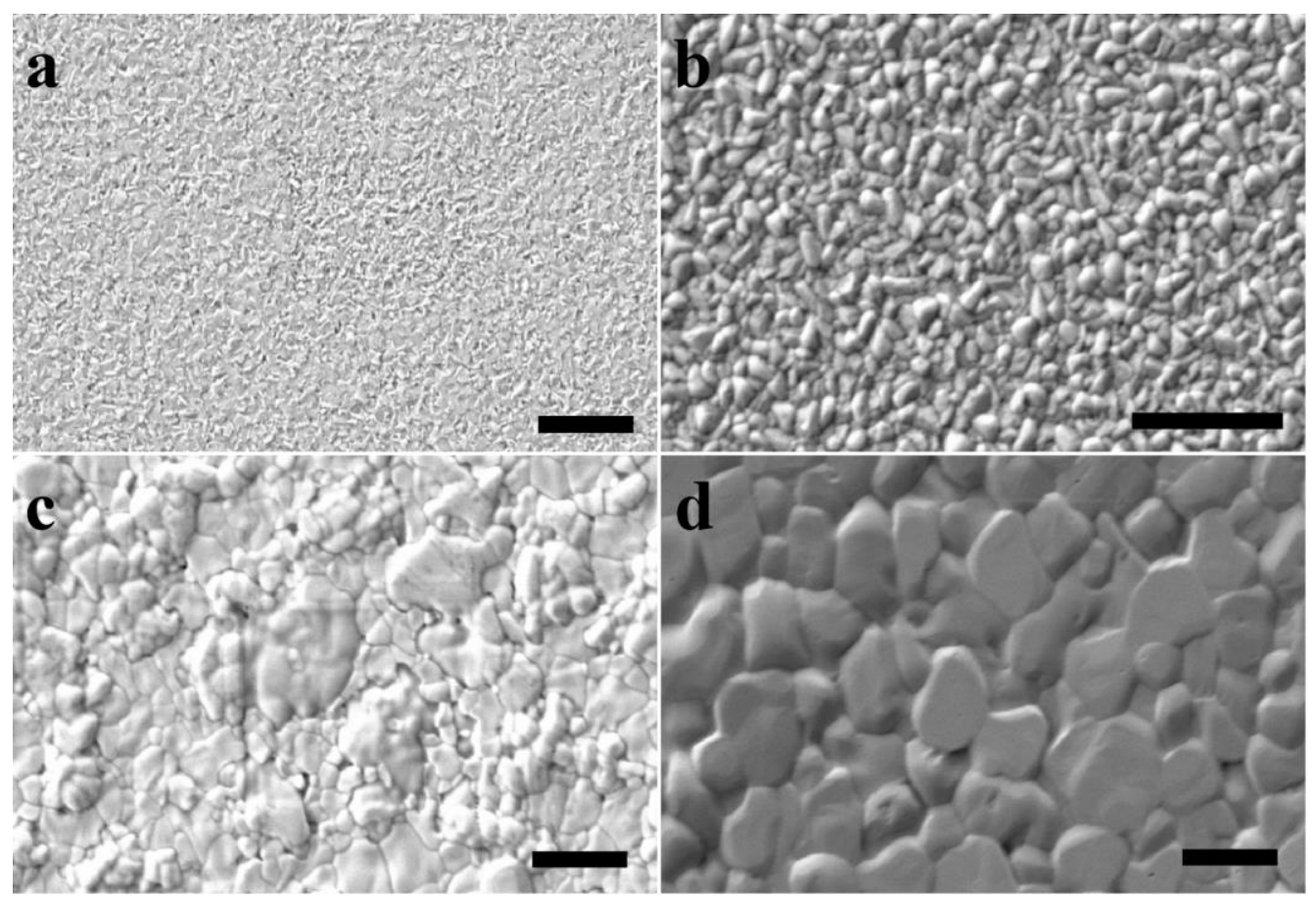

Figure S2: SEM images of co-evaporated MAPI thin films on silicon with varying MA content: (a) $50 \%$ MA, (b) $65 \% \mathrm{MA}$, (c) $80 \% \mathrm{MA}$, and (d) 130\% MA. Scale bars correspond to $1 \mu \mathrm{m}$. 